\title{
Data Management and Three-dimensional Visualization of Global Velocity Model Crust 2.0
}

\author{
Xuefeng Zheng*, Yuwei Sun, and Xiong Xu
}

Northwest Institute of Nuclear Technology, Xi'an, China

*Corresponding author: Xuefeng Zheng, xfzheng@nint.ac.cn

\begin{abstract}
This paper described the method for managing and visualizing three-dimensional earth velocity model Crust 2.0 based on knowledgebase. Firstly, we managed the velocity structure data with Geodatabase. And then, we presented a method to model and visualize the three-dimensional velocity structure with the combination of cuboids and quad-prisms. Furthermore, we have developed a software package to realize the visualization of CRUST2.0, in which the viewing from different directions and the cutting along any path are permitted. Lastly, we have integrated them to the knowledgebase. Based on this, the emulation for seismic wave propagation can be realized.
\end{abstract}

Keywords: three-dimensional visualization, CRUST2.0, openGL, velocity structure

\section{Introduction}

CRUST2.0 is a global crustal model at 2 x 2 degrees for the seismic velocity (Vp and Vs) and density structure. It is a significantly updated version of CRUST 5.1 and takes advantages of a digital sediment map that is defined on a 1x1 degree grid and of still ongoing compilation of new data on crustal structure. With the development of modeling and inversion techniques in seismology, three dimensional earth models, at global or regional scales, are begging to play more and more important roles in both monitoring and research activities related to seismic signals. And to explore advantages of 3D earth models for seismological studies, it is necessary to develop ways for the management and visualization of them.

Many research works have been devoted to this issue in the past decades. But, because of the diversity of data structure of 3D spatial objects, scholars introduced completely distinct ways to meet their respective need. Using the finite element method, F.J. Serón constructed a visualization model for a simple salt dome. Based on the integration environment of Texaco Visualization Center [1], T.M. Sheffield researched the use of colors to represent medium features related to seismic wave propagation [2]. To express the geological body with the information from the borehole, a tri-prism was introduced in 3D stratum modeling by Y. Zhang and S.W. Bai in 2001[3]. However, they only considered the case of a normal tri-prism, in which the arris of the tri-prism are parallel with each other and the end quadrilaterals of the tri-prism are at right angles to the edges. P.G. Cheng improved the tri-prism modeling method by introduced the quasi tri-prism [4], which provided a good solution to overcome the constraints. In these studies, most attention focused on geological application. However, researches for geophysical applications received much less attention.

In this paper, based on analyzing for the CRUST2.0 global crustal structure data, the management method with feature class and the visualization modeling method with the combination of cuboids and quad-prism were presented. Using these methods, we have established a vectorization grid dataset in geodatabase to manage the basic data of CRUST2.0 and a 3D visualization model to express the global velocity structure of it. Furthermore, we have developed a software package to realize the visualization of CRUST2.0, in which the viewing from different directions and the cutting along any path are permitted. 


\section{Data Structure and Data Management for Globle Crustal Model CRUST2.0}

The global model CRUST2.0 divides the global surface into 16200 tiles of $2 \times 2$ degree grids that measure $220 \mathrm{~km}$ by $220 \mathrm{~km}$ at the equator. It is composed of $360 \mathrm{key} 1 \mathrm{~d}$-profiles (the crustal types) where one of these profiles is assigned to each $2 \times 2$ degree cell. Each individual profile is a 7 layer 1D-model with L1-ice, L2water, L3-soft sediments, L4-hard sediments, L5-upper crust, L6-middle crust and L7-lower crust. Parameters VP, VS and rho are given explicitly for these 7 layers as well as the mantle below the Moho. Fig. 1 just shows the actual parameter file, and also other files includes the contribute information for the profiles [5].

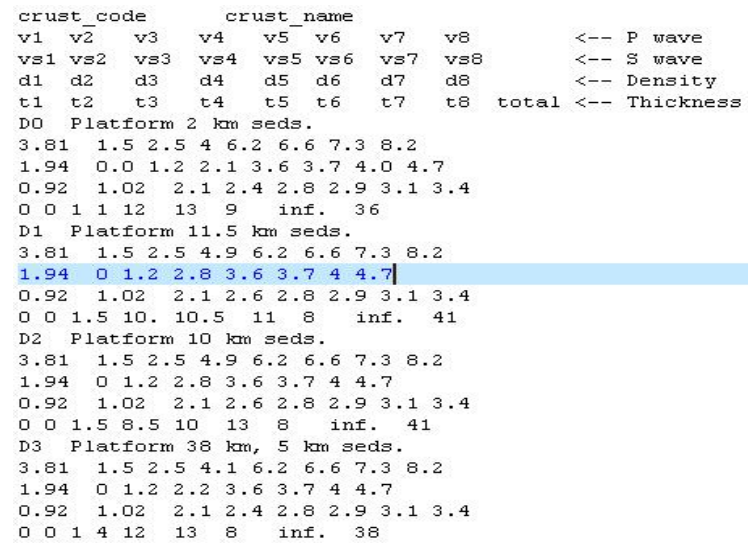

Fig. 1. Model data structure of CRUST2.0

We developed a way to manage the all data for the global model with geodatabase, which includes a feature class for managing the position of 360 key 1d-profiles and two tables for managing the parameters and the relationship each other. Fig. 2 shows the logical structure for global model dataset in the geodatabase. Fig. 3 shows all16200 tiles sharing the 360 key 1d-profiles.
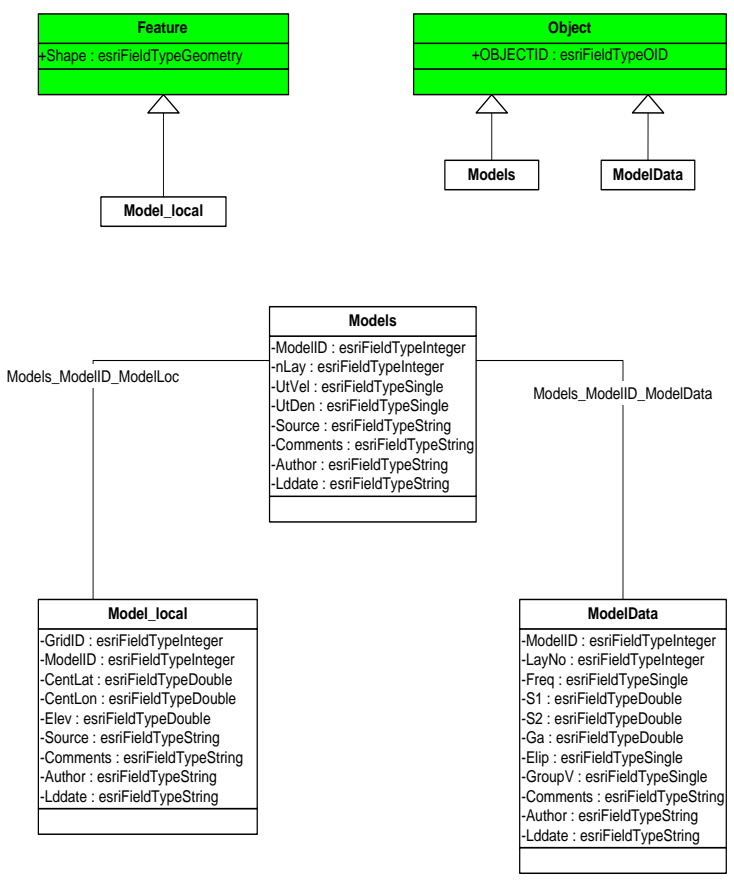

Fig. 2. Global model dataset in the geodatabase 


\section{Data Modeling Method and Visualization Expressing for CRUST2.0}

For the CRUST2.0 model, the data grid, which includes the seismic velocity and density of crustal and uppermost mantle, is regular in the XY plane of the XYZ coordinate space. But it is irregular at the Z direction which accords with the thickness of the layer. Thus, this kind of data volume belongs to the structuralized data,

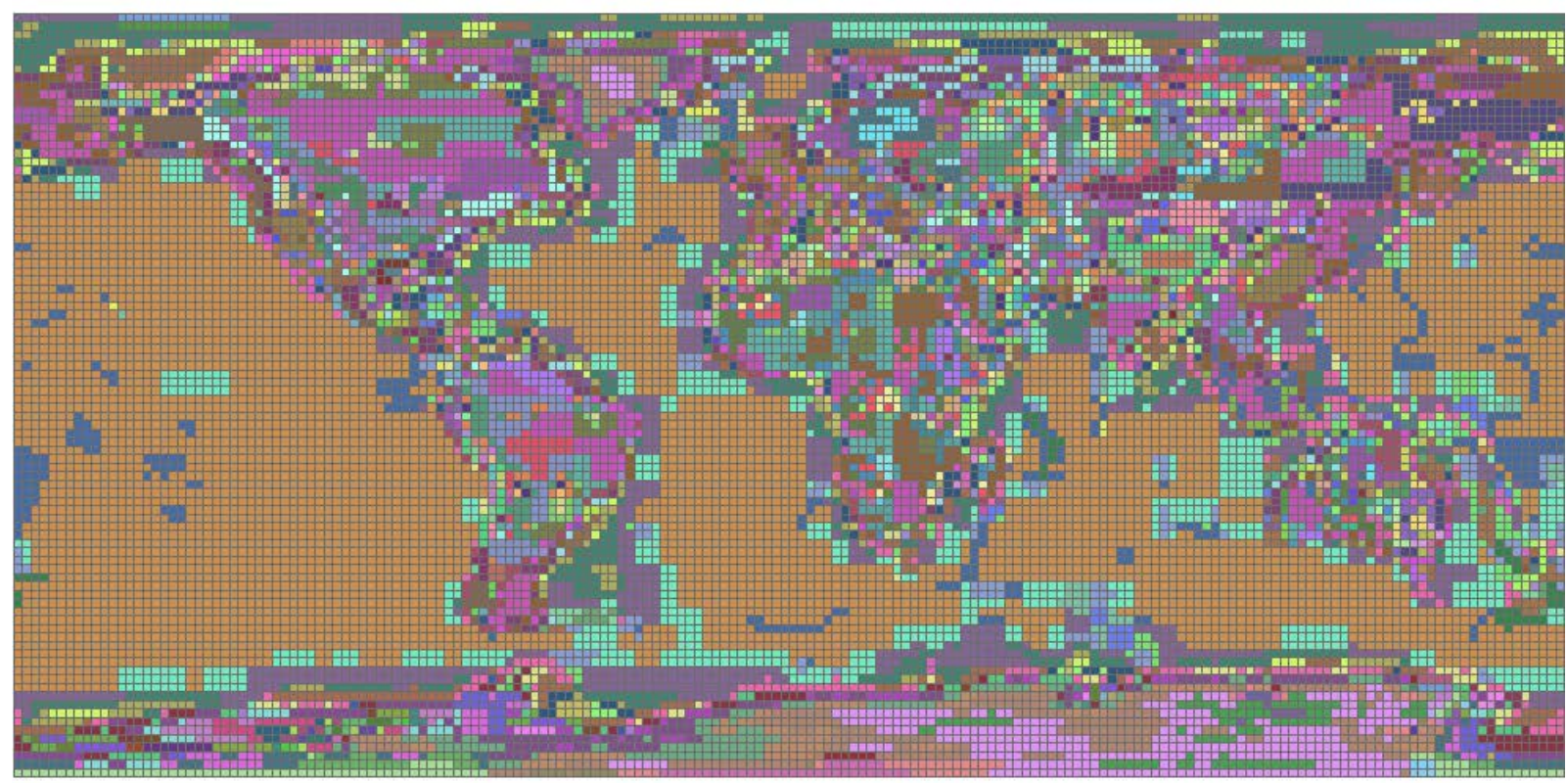

Fig. 3.16200 tiles share 360 seismic velocity and density structures

but not regular grid data sets. It is called rectangle-grid structuralized data sets. In order to describe the data in each grid, the traditional method is to establish the 3D array in which the subscript of 3D array, the data volume and the spatial location must be involved. This data describing method or data structure will require huge amount of computer memory and have high computational complexity.

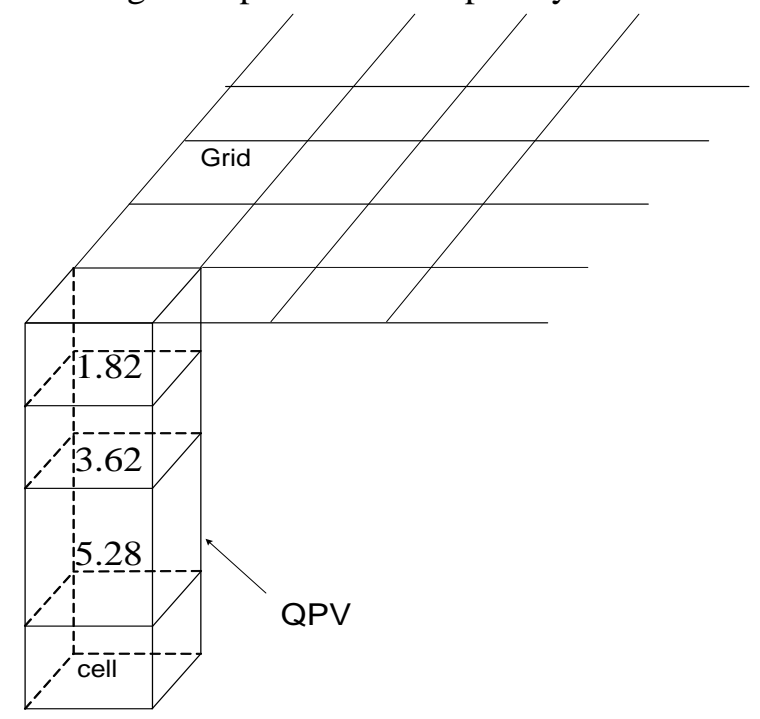

Fig. 4. The 3D grid model based on quad-prism volumes

This paper presents a new visualization modeling method that uses cuboids and quad-prism volume to establish the 3D spatial model of the data sets. In this visualization modeling method, firstly, the Earth surface will be divided into 180*90 (16200) grids that correspond to each tile of CRUST2.0. Each grid from surface to the all crust and uppermost mantle constructs a quad-prism volume (QPV) that can be considered a volume element. 
And then, the quad-prism volume is divided into a group of cuboids according to the numerical value of seismic velocity and density in depth direction, which can be called basic element. Each cuboid is a cell in the visualization model and corresponds only to a group of seismic velocity and density data from one layer of the tile. Thus, the array of quad-prism volumes involved cuboids can express the whole CRUST2.0 model very well. Fig. 4 shows the quad-prism volumes and the gridding.

As above mentioned, the visualization model of CRUST2.0 data based on QPV has been established, but how can we display them in screen and use them in seismological studies? In the 3D visualization field, the OpenGL technique is a kind of important 3D graphic industry standard. It can render the texture features, color the structure volume and express the real world very similarly. In this paper, we developed a software package to display the 3D earth's crust, and establish the basis to simulate the propagation of seismic waves in Delphi with OpenGL. Firstly, we constructed the QPV volume element with the perspective and the texture, and we also constructed the cuboid with the color palette. And then, we established the display list of the QPV according to the tile order. Finally, by painting the scene, the image of visualization model of the CRUST2.0 data was displayed in screen. At the same time, the double-buffer technique has been used in the software to improve the display efficiency.

In order to view the model from different directions and cut it along an arbitrary path, we designed and implemented a rotating and cutting algorithm. The main idea is to confirm and express the visible parts of the QPV and hide the invisible. Fig. 5 shows the visualization model and the cutting and rotating functions.

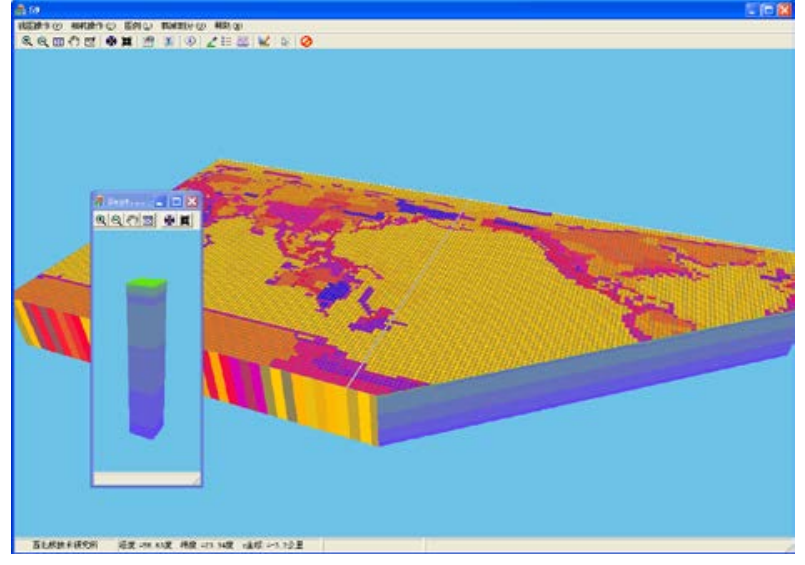

Fig. 5. The visualization model and the cutting and rotating functions

\section{Conclusions}

Based on analyzing for the CRUST2.0 global crustal structure data, the management method with feature class and the visualization modeling method with the combination of cuboids and quad-prism were presented. Using these methods, we have established a vectorization grid dataset in geodatabase to manage the basic data of CRUST2.0 and a 3D visualization model to express the global velocity structure of it. Furthermore, we have developed a software package to realize the visualization of CRUST2.0, in which the viewing from different directions and the cutting along any path are permitted.

\section{References}

1. F.J. Serón, Visualization and finite element techniques for seismic interpretation,Computer \& Graphics in Spain, vol. 16, No.4, pp. 383-394, 1992.

2. T.M. Sheffield, D. Meyer, J. Lees et al. Geovolume visualization interpretation: Color in 3D volumes, The Leading Edge, vol. 18, No. 6, pp. 668-674, 1999. 
3. Y. Zhang, S.W. Bai, An approach of 3D stratum modeling based on tri-prism volume elements,” Journal of Image and Graphics, vol. 6, No. 3, pp. 285-290, 2001.

4. J.Y. Gong, P.G. Cheng, Y.D. Wang, Three-dimensional modeling and application in geological exploration engineering, Computers \& Geosciences, vol. 30, No.4, pp. 391-404, 2004.

5. Gabi Laske, http://mahi.ucsd.edu/Gabi/crust2.html, 2005. 